\title{
The European Legislation Applicable to Medium-Range Inductive Wireless Power Transmission Systems
}

\author{
Frédéric Broydé, ${ }^{1}$ Evelyne Clavelier, ${ }^{1}$ and Lucie Broydé ${ }^{2}$ \\ ${ }^{1}$ Tekcem, 78580 Maule, France \\ ${ }^{2}$ ESIGELEC, Saint-Étienne-du-Rouvray, 76800, France \\ Correspondence should be addressed to Frédéric Broydé; fredbroyde@excem.fr
}

Received 8 January 2014; Revised 6 May 2014; Accepted 11 June 2014; Published 10 July 2014

Academic Editor: Frederick Mailly

Copyright (c) 2014 Frédéric Broydé et al. This is an open access article distributed under the Creative Commons Attribution License, which permits unrestricted use, distribution, and reproduction in any medium, provided the original work is properly cited.

\begin{abstract}
Medium-range inductive wireless power transmission systems allow a sufficient power transfer without requiring close proximity between a primary coil and a secondary coil. We briefly investigate the range of a typical system and its radiated emission, from the perspectives of electromagnetic compatibility (EMC) and human exposure requirements. We then discuss the applicable legislation in the European Union, the main question being the applicability of the R\&TTE or radio equipment directives. Our conclusion is that this applicability depends on multiple parameters, among which is the presence of a self-tuning capability or of a transmitter control based on telemetry.
\end{abstract}

\section{Introduction}

Varying electric fields and magnetic fields can be used for wireless power transmission (WPT) from the antenna of a power-transmitting unit to the antenna of a power-receiving unit. WPT using electrically small coils as antennas is usually referred to as inductive WPT. Here, the antenna used in the power-transmitting unit is a primary coil and the receiving antenna is a secondary coil, as shown in Figure 1. The primary coil and the secondary coil form a transformer having a primary and a secondary which are mechanically separable. When the two are placed in proper orientation and proximity, the coupling becomes sufficient to allow an adequate power transmission.

In a short-range inductive WPT system, power transmission takes place only when the power-transmitting unit and the power-receiving unit have a well-defined position with respect to each other and are in mechanical contact with each other. Thus, the primary and secondary coils are typically a few millimeters apart and a good efficiency can be obtained. Short-range WPT systems are for instance used in chargers for the rechargeable batteries of hand-held battery-operated items, such as electric tooth brushes, radio transceivers, and cellular telephones. This approach allows the hand-held item to be completely sealed and uses no electrical connection between the mains and the hand-held item.

This paper is about medium-range inductive WPT systems, which do not require a close proximity between the primary and secondary coils during power transmission. For instance, an experimental medium-range inductive WPT system (MRIWPTS) was used to power-feed a light bulb over a distance of $2 \mathrm{~m}$ [1-3]. The added convenience of positioningfree operation entails an increased emission level, which can exceed electromagnetic compatibility (EMC) and human exposure requirements (in this paper, human exposure refers to human exposure to electromagnetic fields).

In the European Union (EU), the legislation applicable to an electrical apparatus is set forth in the applicable directive(s), the national legislations transposing the provisions of these directives, and other national legislations. A manufacturer who wants to place a MRIWPTS on the market in the UE must first determine the applicable directive(s), which determine the set of applicable harmonized standards, and the applicable conformity assessment procedures.

The main subject of this paper is a discussion of the directive applicable to a MRIWPTS, the main question being the applicability of the radio equipment and telecommunications terminal equipment (R\&TTE) directive [4] or of 


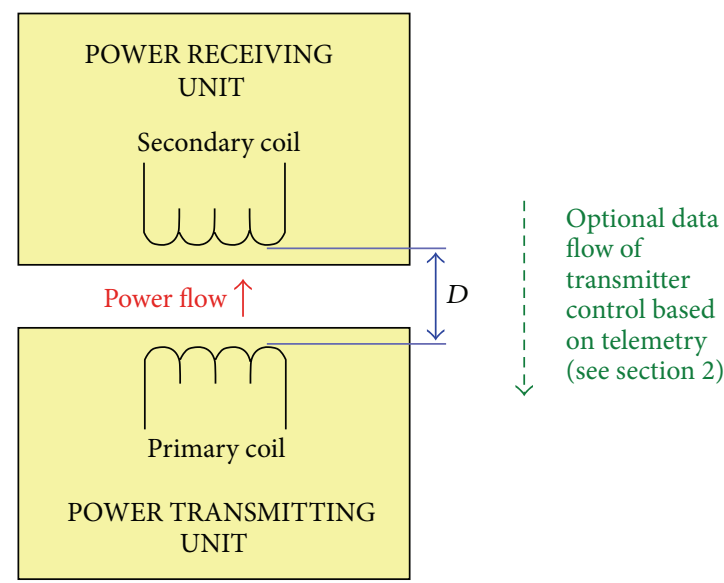

FIgURE 1: An inductive WPT system.

the radio equipment directive which will replace it [5]. If the R\&TTE or radio equipment directive is applicable, it covers electromagnetic compatibility (EMC), as well as safety and protection of health issues, including human exposure. In the opposite case, EMC is usually covered by the EMC directive $[6,7]$, while safety and protection of health, including human exposure, are usually covered by the low voltage directive [ 8 , 9]. Our discussion will not take into account the fact that the MRIWPTS may be a part of a product having other functions influencing the applicability of the R\&TTE directive.

WPT is very useful to power-feed implantable medical devices. It is therefore worth mentioning that a device covered by the medical devices directive [10] or by the active implantable medical devices directive [11] is not subject to the EMC directive and low voltage directive, but it may be subject to the R\&TTE or radio equipment directives.

The paper is organized as follows. Section 2 presents the typical characteristics of inductive WPT systems and provides a structural definition of MRIWPTSs. Section 3 presents a computation of the power gain, which is a measure of the efficiency of a WPT system, as a function of the distance between the coils. Section 4 addresses the radiated emission of medium-range WPT systems. Section 5 explains that one should consider that radio waves are used in WPT. Sections 6 and 7 discuss the applicability of the R\&TTE and radio equipment directives.

\section{Overview of Inductive WPT Systems}

Let us use $D$ to denote the shortest distance between the primary coil and the secondary coil (including their magnetic circuits, if present). Let us use the wording resonant coil to designate a coil used in a series or parallel resonant circuit comprising a capacitor, or a coil used at its self-resonance frequency. An inductive WPT system may use the following:

(i) nonresonant coils in the power-transmitting unit and in the power-receiving unit, in which case a reasonable efficiency requires that a magnetic circuit made of a magnetic material is used to obtain a strong coupling between the coils (i.e., a coefficient of coupling close to 1 ), $D$ being a gap much smaller than the section of the magnetic circuit; (ii) one resonant coil and one nonresonant coil, in which case a reasonable efficiency does not require a magnetic circuit made of a magnetic material, and $D$ may be increased compared to two nonresonant coils;

(iii) resonant coils in the power-transmitting unit and in the power-receiving unit, in which case a reasonable efficiency does not require a magnetic circuit made of a magnetic material, and $D$ may be further increased compared to a device using only one resonant coil, up to a few times the largest dimension of one of the coils.

In current inductive WPT systems, the primary coil is excited by a high-frequency current generated by an electronic circuit, typically a switched-mode or resonant inverter operating at a frequency above $20 \mathrm{kHz}$. Such a design typically implements resonant coils in the power-transmitting unit and/or in the power-receiving unit. For instance,

(i) many transponders for radio frequency identification (RFID) use inductive WPT to supply enough power to sustain the operation of the transponder, typically $10 \mu \mathrm{W}$ to $1 \mathrm{~mW}$ [12];

(ii) implantable medical device typically uses inductive WPT with resonant coils in the frequency range $1 \mathrm{MHz}$ to $20 \mathrm{MHz}$ to deliver $5 \mathrm{~mW}$ to $250 \mathrm{~mW}$ to the implant [13-16];

(iii) a Wireless Power Consortium has issued a specification for inductive WPT systems operating in the $110 \mathrm{kHz}$ to $205 \mathrm{kHz}$ frequency range and capable of delivering up to $5 \mathrm{~W}$ at a distance of about $5 \mathrm{~mm}$ between the primary and secondary coils [17];

(iv) WPT systems intended to be used as battery chargers for electric vehicles are being designed, for instance, a $30 \mathrm{~kW}$ system operating at a distance of $45 \mathrm{~mm}$ [18].

Inductive WPT systems using resonant coils in the power-transmitting unit and in the power-receiving unit provide the best efficiency for a given size of the coils and a given distance between the coils, but they require an accurate tuning. Additionally, power is wasted if the primary coil is excited when the power-receiving unit is not present or does not need to receive power. In order to avoid this situation and to compensate the effect of component tolerances, temperature changes, component drift, and the effect of nearby conducting or magnetic items, it might be desirable to use 


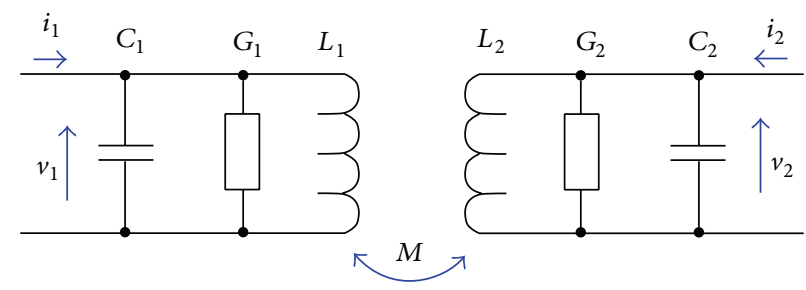

Figure 2: Model for the case of parallel resonance.

a WPT system using a self-tuning capability of the powertransmitting unit or a more elaborate transmitter control based on telemetry involving communication from the powerreceiving unit to the power-transmitting unit. According to the self-tuning capability, the power-transmitting unit is capable of

(i) sensing the presence of a nearby secondary coil connected to a circuit comprising only a capacitor and a resistance, and varying or activating/deactivating the power in the primary coil accordingly; and/or

(ii) dynamically seeking resonance and optimizing power transfer with a nearby secondary coil connected to a circuit comprising only a capacitor and a resistance.

Of course, the self-tuning capability must also be able to operate in the presence of the intended power-receiving unit, which typically comprises a rectifier and other nonlinear circuits. We see that the self-tuning capability helps to obtain a good efficiency.

The above-mentioned specification of the Wireless Power Consortium uses transmitter control based on telemetry, involving a digital communication using load modulation, referred to as "backscatter modulation" in [17]. This specification also uses a form of self-tuning capability, referred to as "analog ping" in [17].

An arbitrary minimum value of $D$ could be used to distinguish a MRIWPTS from a short-range inductive WPT system. However, this approach does not lead to general consequences from the design standpoint. In this paper, a MRIWPTS is defined by a structural attribute: the primary coil does not comprise a magnetic circuit made of a magnetic material.

\section{Power Transfer of Inductive WPT Systems}

We will now assess the typical efficiency which might be expected from a MRIWPTS using resonant coils in the power-transmitting unit and in the power-receiving unit. Since the largest dimension of each coil is small compared to wavelength, we can use a lumped-element circuit model to derive the currents through the coils. We will assume a simple equivalent linear circuit for the power-transmitting unit and the power-receiving unit. This is not a severe limitation since we only want to assess the behavior at the nominal frequency.

We can use the model shown in Figure 2 to investigate a MRIWPTS using a parallel resonant circuit comprising the primary coil, and another parallel resonant circuit comprising the secondary coil. In Figure $2, L_{1}$ is the inductance of the primary coil, $L_{2}$ is the inductance of the secondary coil, and $G_{1}$ and $G_{2}$ are conductances representing the losses in the primary and secondary coils, respectively. $C_{1}$ and $C_{2}$ are the values of the capacitors used to obtain parallel resonance. Since the circuit of the secondary coil is floating, we have not introduced the capacitance between the windings in the model.

We introduce the coefficient of coupling $k$, the quality factors $Q_{1}$ and $Q_{2}$, and the radian frequencies $\omega_{01}$ and $\omega_{02}$, given by

$$
\begin{gathered}
k=\frac{M}{\sqrt{L_{1} L_{2}}}, \\
Q_{1}=\frac{1}{G_{1}} \sqrt{\frac{C_{1}}{L_{1}}}, \quad Q_{2}=\frac{1}{G_{2}} \sqrt{\frac{C_{2}}{L_{2}}}, \\
\omega_{01}=\frac{1}{\sqrt{C_{1} L_{1}}}, \quad \omega_{02}=\frac{1}{\sqrt{C_{2} L_{2}}} .
\end{gathered}
$$

If we consider the problem for which $i_{2}$ is determined by a load of conductance $G_{2 L}$, in such a way that $i_{2}=-G_{2 L} v_{2}$, we define the power gain as

$$
a_{P}=\frac{\operatorname{Re}\left(v_{2} \bar{i}_{2}\right)}{\operatorname{Re}\left(v_{1} \bar{i}_{1}\right)},
$$

where the bar denotes the complex conjugate. A simple analysis shows that if the circuits are tuned to the same frequency $\omega_{0}$, that is, $\omega_{0}=\omega_{01}=\omega_{02}$, and that the coupling is weak, that is, $k \ll 1$, an acceptable efficiency is only obtained for $Q_{1} \gg 1$ and $Q_{2} \gg 1$. Using these assumptions, we find that [19], at $\omega=\omega_{0}$,

$$
a_{P} \approx \frac{K^{2} G_{2} G_{2 L}}{\left(G_{2 L}+G_{2}\left[K^{2}+1\right]\right)\left(G_{2 L}+G_{2}\right)}
$$

in which, following Terman [20, Sections 3 and 5] and Hochmair [13], we have used the dimensionless coupling parameter $K$ defined by

$$
K=k \sqrt{Q_{1} Q_{2}} .
$$

In order to maximize the approximate power gain given by (5) for $G_{2}$ and $K$ fixed, we must use $G_{2 L}=G_{2 L \text { MAX }}$ with

$$
G_{2 L \operatorname{MAX}}=G_{2} \sqrt{1+K^{2}} \text {. }
$$


Figure 3 shows the coefficient of coupling $k$ and the exact power gain $a_{P}$ as a function of the distance $D$ between the primary and secondary coils in a coaxial configuration, for $Q_{1}=Q_{2}=100$ and parallel resonance at the frequency $f_{0}=$ 27.120 MHz. This computation considers 2 identical circular coils of mean radius $r=0.1 \mathrm{~m}$ each having a single turn and no magnetic core. We assume that, at each $D$, the powerreceiving unit absorbs a sinusoidal current and adjusts $G_{2 L}$ so that it takes on the value given by (7).

We can use the model shown in Figure 4 to investigate a MRIWPTS using a series resonant circuit comprising the primary coil $L_{1}$ and a series resonant circuit comprising the secondary coil $L_{2}$. Again, we have not introduced the capacitance between the windings in the model. This WPT system requires a separate analysis because, contrary to appearances, the circuits shown in Figures 2 and 4 are not dually related. In Figure $4, R_{1}$ and $R_{2}$ are resistances representing the losses in the primary and secondary coils, respectively. $C_{1}$ and $C_{2}$ are the values of the capacitors used to obtain series resonance.

We introduce the quality factors $Q_{1}$ and $Q_{2}$ given by

$$
Q_{1}=\frac{1}{R_{1}} \sqrt{\frac{L_{1}}{C_{1}}}, \quad Q_{2}=\frac{1}{R_{2}} \sqrt{\frac{L_{2}}{C_{2}}},
$$

and we will use again $\omega_{01}$ and $\omega_{02}$ given by (3). If we consider the problem for which $i_{2}$ is determined by a load of resistance $R_{2 L}$, in such a way that $v_{2}=-R_{2 L} i_{2}$, we may use the power gain given by (4). In practice, as above, we can assume that the circuits are tuned to the same frequency $\omega_{0}=\omega_{01}=\omega_{02}$, and we find that [19], at $\omega=\omega_{0}$,

$$
a_{P}=\frac{K^{2} R_{2} R_{2 L}}{\left(R_{2 L}+R_{2}\left[K^{2}+1\right]\right)\left(R_{2 L}+R_{2}\right)} .
$$

In order to maximize the power gain for $R_{2}$ and $K$ fixed, we must use $R_{2 L}=R_{2 L \text { MAX }}$ with

$$
R_{2 L \operatorname{MAX}}=R_{2} \sqrt{1+K^{2}} \text {. }
$$

We can compute the power gain $a_{P}$ as a function of the distance $D$ between the primary and secondary coils, for the geometry and the coils defined above in the explanations for Figure 3, using series resonance at the frequency $f_{0}=$ 27.120 MHz. Assuming that, at each $D$, the power-receiving unit absorbs a sinusoidal current and adjusts $R_{2 L}$ so that it takes on the value given by (10), the exact value of the power gain $a_{P}$ differs by less than $0.5 \%$ from the results obtained for parallel resonance. Thus, Figure 3 also applies to series resonance.

The results presented above are very similar to the ones presented in [19] for a design having a similar geometry but operating at $f_{0}=149 \mathrm{kHz}$. To obtain a reasonable efficiency, the ratio of the shortest distance between the coils to the largest dimension of the largest coil will always be less than 4.0 in an ideal experiment.

There are two other simple configurations which may be used to obtain resonant coils in the power-transmitting unit and in the power-receiving unit: parallel resonance in the power-transmitting unit with series resonance in the powerreceiving unit and vice versa [18]. The latter seems to be the most popular scheme. However, more complex structures can also be used, for instance, involving two coupled coils in the power-transmitting unit and in the power-receiving unit [2], or a dual resonant circuit $[16,17]$.

\section{Emission of a MRIWPTS}

We now want to determine the radiated emission of the MRIWPTS, to investigate EMC and human exposure. This emission is mainly characterized by the magnetic field intensity $H$, which will be computed in free space. We assume that the excitation of the resonant circuit comprising the primary coil is adjusted in such a way that a power of $1 \mathrm{~W}$ is delivered to the load of the power-receiving unit, in addition to the earlier assumption that the power-receiving unit absorbs the maximum power from the resonant circuit comprising the secondary coil.

If we measure $H$ at a distance $d$ of the primary coil, $d$ being much larger than $D$ and the largest dimension of the primary and secondary coils, the free space emission is close to the emission of a magnetic dipole, for which the total radiated power $\mathscr{W}$ is given by Stratton [21, page 438]:

$$
\mathscr{W}=\frac{k^{4} \eta_{0}}{6 \pi}|\mathscr{M}|^{2},
$$

where $\mathscr{M}$ is the r.m.s. magnetic moment of the dipole, $\eta_{0} \approx$ $376.7 \Omega$ is the intrinsic impedance of free space, and $k$ is the wave number $\omega / c_{0}$ where $c_{0}$ is the velocity of light in free space. For the configuration used above, comprising two coaxial circular coils, we have

$$
|\mathscr{M}|=\pi r^{2}\left(i_{L 1}+i_{L 2}\right)
$$

where $i_{L 1}$ and $i_{L 2}$ are the r.m.s. currents flowing in the primary coil and in the secondary coil, respectively.

Figure 5 shows $\mathscr{W}$ computed as a function of $D$, in the case of the configurations shown in Figures 2 and 4. In the case of parallel resonance shown in Figure $2, i_{L 1}$ is the sum of the currents flowing through $L_{1}$ and $G_{1}$, and $i_{L 2}$ is the sum of the currents flowing through $L_{2}$ and $G_{2}$. In the case of series resonance shown in Figure 4 , we have $i_{L 1}=i_{1}$ and $i_{L 2}=i_{2}$. Once $\mathscr{W}$ is computed, the maximum r.m.s. magnetic field strength at the distance $d$, denoted by $H_{\mathrm{MAX}}$, is given by Broydé and Clavelier [22]:

$$
H_{\mathrm{MAX}}=\frac{1}{d} \sqrt{\frac{3 \mathscr{W}}{8 \pi \eta_{0}}} \frac{\ell}{(k d)^{2}},
$$

where

$$
\begin{gathered}
f= \begin{cases}2 \sqrt{(k d)^{2}+1} & \text { if } k d \leq(k d)_{C} \\
\sqrt{(k d)^{4}-(k d)^{2}+1} & \text { if } k d>(k d)_{C}\end{cases} \\
(k d)_{C} \equiv \sqrt{\frac{5+\sqrt{37}}{2}} \approx 2.354 .
\end{gathered}
$$




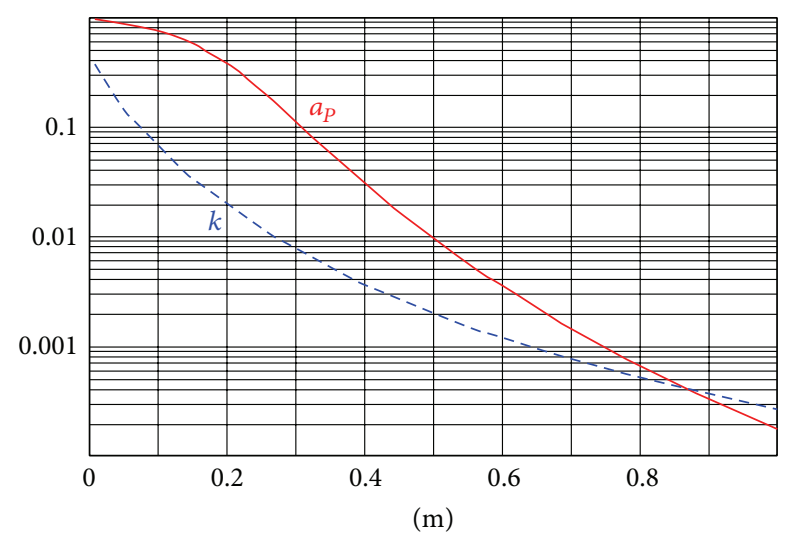

FIgURE 3: Coefficient of coupling $k$ and power gain $a_{P}$ versus $D$.

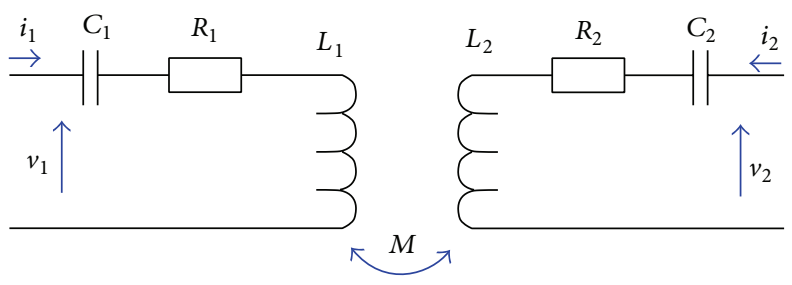

FIGURE 4: Model for the case of series resonance.

The field strength given by (13)-(15) is exact for a magnetic dipole at any frequency, in which case $d$ is the distance between the dipole and the point where the field is measured. It applies to our problem only if the distance $d$ between the center of the primary coil and the measurement point is much larger than $D$ and the largest dimension of the primary and secondary coils. At a distance $d$ which is not much larger than $D$ and the largest dimension of the primary and secondary coils, we need to compute $H$ at each point in space. Figure 6 shows a map of $H$, in the case of the configuration shown in Figure 2, for coaxial coils and $D=$ $0.3 \mathrm{~m}$. Here, we have $D / 2 r=1.5$ and $a_{P}=0.163$. To obtain this map, we have used the exact analytical formula for the field intensity produced by each circular loop in the near-field region [21, p. 263, Problem 4]. The reference level relating to the limitation of exposure of the general public defined in [23] at the frequency $f_{0}=27.120 \mathrm{MHz}$, namely, $73 \mathrm{~mA} / \mathrm{m}$ or $97.27 \mathrm{~dB}(\mu \mathrm{A} / \mathrm{m})$, is $0 \mathrm{~dB}$ in Figure 6. Figure 7 shows $H$, expressed in $\mathrm{dB}(\mu \mathrm{A} / \mathrm{m})$, for $D=0.3 \mathrm{~m}$, as a function of the coordinate $z$ along the axis, the center of the primary lying at $z=0 \mathrm{~m}$ and the center of the secondary lying at $z=0.3 \mathrm{~m}$. In Figure 7, the solid curve is the result of the exact near-field computation used to obtain Figure 6, and the dashed curve is obtained using the exact magnetic dipole formulas (13)(15). We note that, in Figures 6 and 7, the field strength is approximately as high near the secondary coil as it is near the primary coil.

According to Figure 5, the radiated emission of the MRIWPTS computed here is much higher than the one obtained in [19] at $f_{0}=149 \mathrm{kHz}$. For instance, for $D=1 \mathrm{~m}$, the total radiated power is about $40 \mathrm{dBm}$ in Figure 5 (at $f_{0}$ $=27.120 \mathrm{MHz}$ ), as opposed to only $-30 \mathrm{dBm}$ in [19] (at $f_{0}=$ $149 \mathrm{kHz}$ ). With regard to human exposure, the field levels are much lower in Figure 6 than in the corresponding map of [19], but the reference levels defined in [23] are also different: $73 \mathrm{~mA} / \mathrm{m}$ at $f_{0}=27.120 \mathrm{MHz}$ versus $5 \mathrm{~A} / \mathrm{m}$ at $f_{0}=149 \mathrm{kHz}$. The bottom line is that the volume where the reference level relating to the limitation of exposure of the general public is exceeded, which corresponds to the interior of the $0 \mathrm{~dB}$ curve in Figure 6, is larger in the case of the $27.120 \mathrm{MHz}$ design studied in this paper than it is for the $149 \mathrm{kHz}$ design.

Figure 8 shows a WPT experiment corresponding to the configuration considered in Figures 6 and 7: two single-turn coils of radius $0.1 \mathrm{~m}$ separated by a distance $D=0.3 \mathrm{~m}$ are used at $27.120 \mathrm{MHz}$. The secondary coil is suspended by a thin string and connected to a $110 \mathrm{~V}$ neon lamp in parallel with an air dielectric adjustable capacitor. In this simple experiment, the transmitted power is used to power-feed the lamp, and the power received by the lamp is not measured because the lamp is highly nonlinear. The primary coil is connected to the antenna port of an antenna tuner which receives about $6 \mathrm{~W}$ at its transmitter port. The hand of one of the authors is placed between the coils, no effect of the field being perceived. The lamp was then replaced with a resistor of $13.35 \mathrm{k} \Omega$ (measured value) and the power received by the transmitter port of the antenna tuner was set to about $48 \mathrm{~W}$, to obtain a power received by the resistor equal to $1 \mathrm{~W}$. An $\mathrm{H}$-field probe was inserted between the coils, at $z=0.15 \mathrm{~m}$. This caused a reduction of the power received by the resistor, which was not compensated by any new adjustment. The field 


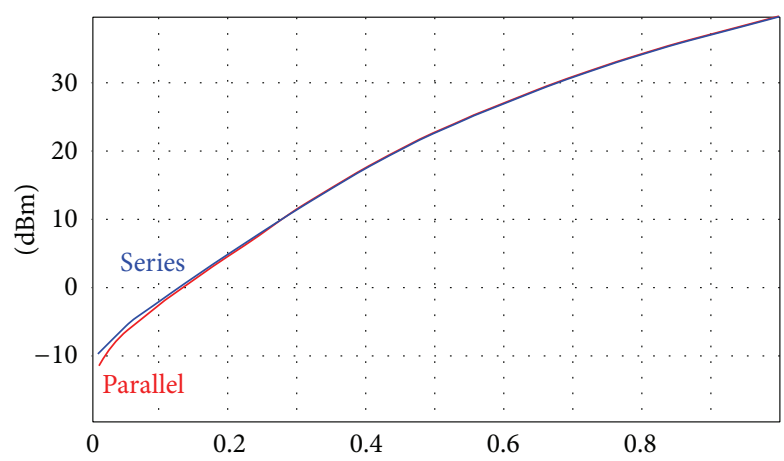

$(\mathrm{m})$

FIgURE 5: Total radiated power $\mathscr{W}$ as a function of the distance $D$ between the coils, for $1 \mathrm{~W}$ delivered to the load.

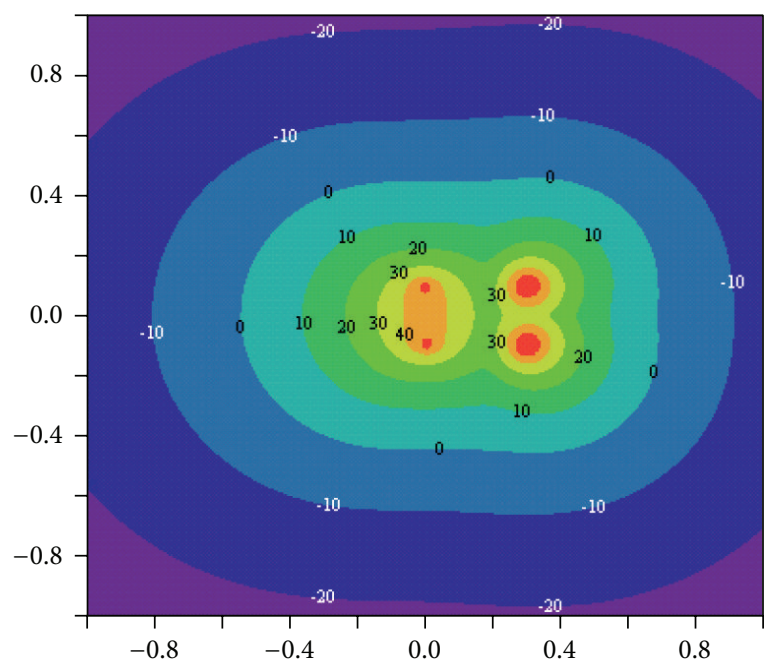

Figure 6: Map of $H$ in a plane containing the common axis of the coils, for $D=0.3 \mathrm{~m}$. The origin is the center of the primary coil. Horizontal and vertical axes: cartesian coordinates in meters. Colors in $10 \mathrm{~dB}$ steps, $0 \mathrm{~dB}$ corresponding to about $97.27 \mathrm{~dB}(\mu \mathrm{A} / \mathrm{m})$ or $0.073 \mathrm{~A} / \mathrm{m}$.

between the coils was measured to be about $126 \mathrm{~dB}(\mu \mathrm{A} / \mathrm{m})$. The agreement with Figure 7 is acceptable if we consider that the actual values of $Q_{1}$ and $Q_{2}$ are not known and that the $\mathrm{H}$-field probe disturbs the field, as evidenced by the received power variation.

If we assume that a MRIWPTS is subject to the R\&TTE directive, we may have to use the standards applicable to inductive loop systems in the frequency range $9 \mathrm{kHz}$ to $30 \mathrm{MHz}$, under this directive $[24,25]$. In this case, for the MRIWPTS at $f_{0}=27.120 \mathrm{MHz}$, the radiated emission limit is $42 \mathrm{~dB}(\mu \mathrm{A} / \mathrm{m})$, measured at $10 \mathrm{~m}$. If we assume $D=0.3 \mathrm{~m}$ and a power of $1 \mathrm{~W}$ delivered to the load of the power-receiving unit, as in Figure 6 to Figure 8, the total radiated power is about $11 \mathrm{dBm}$ in Figure 5, for which the field value given by the exact magnetic dipole formulas (13)-(15) at $10 \mathrm{~m}$ is about $46 \mathrm{~dB}(\mu \mathrm{A} / \mathrm{m})$. The emission limit is exceeded by about $4 \mathrm{~dB}$ so that the design must be revised.

If we assume that a MRIWPTS is subject to the EMC directive, we may, for instance, be allowed to use the EN 55011 standards [26]. In this case, for the MRIWPTS at $f_{0}=$ $27.120 \mathrm{MHz}$, no radiated emission limit is applicable because an ISM frequency is used. Here, we only have to take into account human exposure requirements.

In this section we have had a closer look at two key parameters of a MRIWPTS, the total radiated power and the magnetic field distribution close to the coils, which are dependent on the distance $D$. We have seen that the applicable technical requirements regarding radiated emission and human exposure play an essential role in an actual design. This is why it is important to correctly determine the applicable legislation.

\section{Inductive WPT and Electromagnetic Waves}

The term radio wave is defined in Article 2 of the R\&TTE directive, as a special case of electromagnetic wave. Unfortunately, electromagnetic wave is neither defined in the directive, nor unambiguously defined in the literature. The generally accepted definition in textbooks on electromagnetic theory is that electromagnetic waves are the vectors $\mathbf{E}$ and $\mathbf{H}$ solutions of the vector wave equation [21, Section 7.1]. Any 


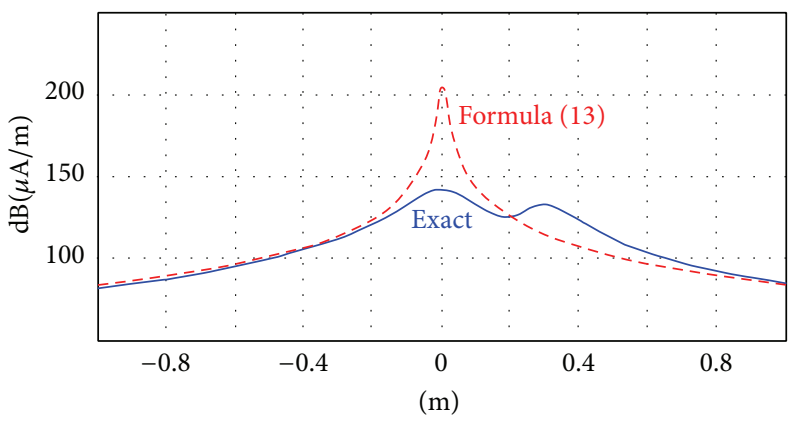

FIgURE 7: $H$ along the common axis of the coils, for $D=0.3 \mathrm{~m}$. The origin is the center of the primary coil.

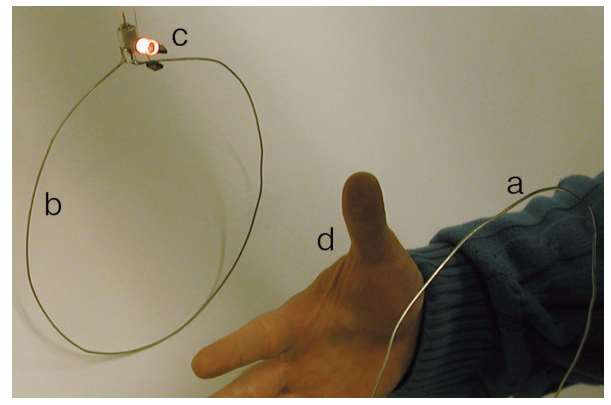

FIGURE 8: A WPT experiment according to the configuration considered in the paper, for $D=0.3 \mathrm{~m}$, at $27.120 \mathrm{MHz}$ : (a) is a part of the primary coil, (b) is the secondary coil, (c) is a neon lamp connected in parallel with the secondary coil, and (d) is an experimenter's hand. The lamp is being lit by wireless power.

such electromagnetic wave propagates. This concept is very general: all field distributions are included, except static ones.

This question of vocabulary is not academic since, depending on the meaning given to "electromagnetic waves," different European directives may be applicable, which entail different requirements and different costs (engineering, conformity assessment, and manufacturing costs). This may be why the wording nonradiative, which suggests that electromagnetic waves are not generated, has been associated by some authors to their wireless power transfer concepts [3, 27].

The particular fields produced by a harmonic electric or magnetic dipole can be written as the sum of three terms: the "quasistatic" term in $1 / r^{3}$, the "induction" term in $1 / r^{2}$, and the "radiation" term in $1 / r$ [21, Sections 8.5, and 8.6], where $r$ denotes the distance between the dipole and the measurement point. For instance, in the case of a magnetic dipole of particular interest in this paper, if we assume and suppress a time-harmonic dependence $e^{j \omega t}$, the magnetic field applied by the magnetic dipole at a location defined by the vector $\mathbf{r}$ is given by

$$
\begin{gathered}
\mathbf{H}=\frac{e^{-j k r}}{4 \pi}\left\{\left(\frac{1}{r^{3}}+\frac{j k}{r^{2}}\right)[3(\mathbf{u} \cdot \mathbf{M}) \mathbf{u}-\mathbf{M}]\right. \\
\left.-\frac{k^{2}}{r}[\mathbf{u} \times(\mathbf{u} \times \mathbf{M})]\right\},
\end{gathered}
$$

where $\mathbf{u}$ is a unit vector in the direction of $\mathbf{r}$, such that $\mathbf{r}=r \mathbf{u}$, where $k$ is the wave number and where $\mathbf{M}$ is the magnetic moment vector. At a distance from the electric or magnetic dipole less than $\lambda / 2 \pi$, where $\lambda$ is the wavelength, $\ell r$ is less than 1 so that the "quasi-static" term predominates. Technically, the three terms propagate, because they contain the propagation factor $e^{-j k r}$ which indicates the propagation of a spherical wave at the phase velocity $c_{0}$.

More generally, if we consider an arbitrary timeharmonic source of variable electric and magnetic fields, the total field at any point is a volume integral of several terms, each term propagating everywhere [21, Section 8.14] [28, Section 7.9]. For instance, the magnetic field applied by the source is given by

$$
\mathbf{H}=\frac{1}{4 \pi} \iint_{\text {Source }} \int_{r^{2}}\left(\frac{1}{r}\right) \mathrm{e}^{-j k r} \mathbf{u} \times \mathbf{j}_{T} d V,
$$

where $\mathbf{j}_{T}$ denotes the current density from the free charges and the polarization charges. Here, the contribution of a volume element $d V$ has a term in $1 / r^{2}$ and a term in $1 / r$, and both propagate at the velocity of light in free space. Thus, propagation takes place everywhere, in the far-field region and in the near-field region of the source. Thus, we should not be misled by the word "static": in the region where the field is quasistatic, the field can be approximately computed as a static field, but the field nevertheless propagates.

Definitions being arbitrary, different points of view are of course possible; hence, the wording "nor unambiguously defined" is used at the beginning of this section. We note that the perspective of elementary explanations on electromagnetic waves, which mostly focus on plane waves and 
what happens in the far-field region of an antenna where the radiation term dominates, suggests that electromagnetic wave and propagation only exist far from the sources. As shown above, this is misleading. This perspective does not lead to a general definition of "electromagnetic wave."

Engineers do not usually consider capacitors, inductors, or transformers as devices in which electromagnetic waves occur. This is because these circuit elements are usually introduced in circuit theory without reference to the more general framework of electromagnetic theory. This is also because electromagnetic propagation is not needed to explain their basic properties. However, we must also face the fact that if we consider electromagnetic wave in every sense of the word, the coupling between the primary coil and the secondary coil of a MRIWPTS is caused by electromagnetic waves.

\section{Applicability of the R\&TTE Directive}

The R\&TTE directive, which will remain applicable until 12 June 2016 [5, Art. 50], is applicable to a radio equipment, defined as a product or component capable of communication by means of the emission and/or the reception of radio waves, where radio waves mean electromagnetic waves of frequencies from $9 \mathrm{kHz}$ to $3000 \mathrm{GHz}$, propagated in space without artificial guide.

The fact that, in the meaning of the R\&TTE directive, inductive coupling uses electromagnetic waves cannot be questioned, since harmonized standards exist for the application of the R\&TTE directive to inductive loop systems $[24,25]$. This confirms the analysis of Section 5.

Let us now have a closer look at the concept of electromagnetic waves propagated in space without artificial guide. In this requirement, "propagated in space without artificial guide" obviously refers to the capability of communication covering a sufficient distance in any direction, because this capability implies that interactions with other items capable of emission and/or reception of electromagnetic waves cannot be controlled by the user of the product or other interested parties, as opposed to transmission through an electrical interconnection. In other words, if the range is too short, the capability of communicating with electromagnetic waves should be ignored. This is one of the consequences of the wording "in space without artificial guide": there must be enough space (i.e., empty gap).

No such minimum distance appears in the R\&TTE directive. However, the current "Guide to the R\&TTE Directive 1999/5/EC" excludes cochlear implants of the "current technology" from the scope of the R\&TTE directive [29, Section 1.3.5], on the basis of a report prepared by the authors, which has been made public by the European Commission [30]. According to this report, a product comprising a transmitter and a receiver could possibly not be regarded as being in the scope of the R\&TTE directive, when the following conditions are simultaneously fulfilled:

(a) the receiver is intended to operate, at a given time, with a single transmitter of the intended type of transmitter, which we will refer to as "the intended transmitter"; (b) the receiver has a maximum range below $10 \mathrm{~cm}$ for the intended type of transmitter so that, in a given configuration, the intended transmitter is the only transmitter of the intended type capable of communication with the receiver, as if the transmitted signal was sent through an electrical interconnection;

(c) the receiver is so insensitive that it cannot, in normal electromagnetic environments, receive electromagnetic waves coming from other sources than the intended transmitter.

Any practical MRIWPTS is likely to operate at frequencies higher than $9 \mathrm{kHz}$ for power transmission. At this stage, we may conclude that such a MRIWPTS uses radio waves unless it meets the conditions (a), (b), and (c) defined above.

To determine in which case a MRIWPTS is radio equipment subject to the R\&TTE directive, we must now discuss whether a MRIWPTS is capable of communication. At first glance, it seems that the electromagnetic waves used for power transmission do not convey any information and that no communication is therefore taking place. However, we must also consider the possible implementation of a selftuning capability of the power-transmitting unit or a more elaborate transmitter control based on telemetry, as explained in Section 2.

An accepted interpretation of the R\&TTE directive concerning radars states that radars are capable of communication. The rationale for this interpretation says that it results from the assumption that "communication is considered merely the act of transmitting or receiving signals." Presumably, such transmitted or received signal is implicitly modulated so as to convey information. This interpretation is in line with the fact that the International Telecommunication Union (ITU) covers radiolocation (radars). We must therefore consider that a MRIWPTS using transmitter control based on telemetry is capable of communication so that it falls within the scope of the R\&TTE directive if it operates at frequencies higher than $9 \mathrm{kHz}$ for power transmission and if it does not meet the conditions (a), (b), and (c).

The self-tuning capability does not involve modulation conveying information so that we may consider that the self-tuning capability does not imply that a MRIWPTS is capable of communication. Of course, a WPT without selftuning capability or transmitter control based on telemetry involves no modulation conveying information. Thus, we may conclude that a MRIWPTS which does not use transmitter control based on telemetry need not be subject to the R\&TTE directive and that, if it is not capable of radio communication for another purpose than the one discussed here, it does not fall within the scope of the R\&TTE directive.

\section{Applicability of the Radio Equipment Directive}

The radio equipment directive, which will replace the R\&TTE directive on 13 June 2016 [5, Art. 50], uses different definitions. It will be applicable to radio equipment, defined as an electrical or electronic product, which alone or completed with an accessory such as antenna intentionally emits and/or 
receives radio waves for the purpose of radio communication and/or radiodetermination, where radio communication means communication by means of radio waves, radiodetermination means the determination of the position, velocity, and/or other characteristics of an object or the obtaining of information relating to those parameters, by means of the propagation properties of radio waves, and radio waves mean electromagnetic waves of frequencies lower than $3000 \mathrm{GHz}$, propagated in space without artificial guide.

In the radio equipment directive, the minimum frequency of $9 \mathrm{kHz}$ has disappeared from the definition of radio waves, so that a MRIWPTS uses radio waves unless it meets the conditions (a) (b) and (c) defined in Section 4.

At this point, we do not need to discuss the communication capabilities of the MRIWPTS, because the self-tuning capability and transmitter control based on telemetry are clearly intended for radio determination. Thus a MRIWPTS using the self-tuning capability or transmitter control based on telemetry falls within the scope of the radio equipment directive if it does not meet the conditions (a), (b), and (c).

We may also conclude that if a MRIWPTS uses neither a self-tuning capability nor transmitter control based on telemetry, it need not be subject to the radio equipment directive, and that, if it is not capable of radio communication for another purpose than the one discussed here, it does not fall within the scope of the radio equipment directive.

\section{Conclusion}

For a MRIWPTS providing a reasonable efficiency, the ratio of the shortest distance between the coils to the largest dimension of the largest coil will always be less than 4,0 in an ideal experiment and less than 2,0 in a real-world application. For a MRIWPTS operating at the $27.120 \mathrm{MHz}$ frequency, a transmitted power of only $1 \mathrm{~W}$, and a distance of $0.3 \mathrm{~m}$ between the coils, we have shown that a radiated emission limit may or may not be exceeded, depending on the applicable legislation. We have also shown that, for this MRIWPTS, the reference levels relating to the limitation of exposure of the general public may be exceeded near the coils.

We have presented an analysis of the applicability of the R\&TTE and radio equipment directives. The result of this analysis can be summarized into the following simplified statements:

(i) a MRIWPTS falls within the scope of the R\&TTE directive only if it uses transmitter control based on telemetry with a range exceeding $10 \mathrm{~cm}$ or another form of radio communication subject to the R\&TTE directive;

(ii) a MRIWPTS will be subject to the radio equipment directive only if it uses the self-tuning capability or transmitter control based on telemetry, with a range exceeding $10 \mathrm{~cm}$, or another form of radio communication subject to the radio equipment directive.

\section{Conflict of Interests}

The authors declare that there is no conflict of interests regarding the publishing of this paper.

\section{References}

[1] International Patent Application Number PCT/US2006/ 026480 of 5 July 2006 (WO 2007/008646), "Wireless nonradiative energy transfer," Inventors: Joannopoulos et al.

[2] A. Kurs, A. Karalis, R. Moffatt, D. J. Joannopoulos, P. Fisher, and M. Sojacic, "Wireless power transfer via strongly coupled magnetic resonnances," Science, vol. 317, pp. 83-86, 2007.

[3] A. Karalis, J. D. Joannopoulos, and M. Soljačić, "Efficient wireless non-radiative mid-range energy transfer," Annals of Physics, vol. 323, no. 1, pp. 34-48, 2008.

[4] Directive 1999/5/EC of the European Parliament and of the Council of 9 March 1999 on radio equipment and telecommunications terminal equipment and the mutual recognition of their conformity.

[5] Directive 2014/53/EU of the European Parliament and of the Council of 16 April 2014 on the harmonisation of the laws of the Member States relating to the making available on the market of radio equipment and repealing Directive 1999/5/EC.

[6] Directive 2004/108/EC of the European Parliament and of the Council of 15 December 2004 on the approximation of the laws of the Member States relating to electromagnetic compatibility and repealing Directive 89/336/EEC.

[7] Directive 2014/30/EU of the European Parliament and of the Council of 26 February 2014 on the harmonisation of the laws of the Member States relating to electromagnetic compatibility (recast).

[8] Directive 2006/95/EC of the European Parliament and of the Council of 12 December 2006 on the harmonization of the laws of Member States relating to electrical equipment designed for use within certain voltage limits.

[9] Directive 2014/35/EU of the European Parliament and of the Council of 26 February 2014 on the harmonisation of the laws of the Member States relating to the making available on the market of electrical equipment designed for use within certain voltage limits.

[10] Council Directive 93/42/EEC of 14 June 1993 concerning medical devices.

[11] Council Directive 90/385/EEC of 20 June 1990 on the approximation of the laws of the Member States relating to active implantable medical devices.

[12] R. Want, "An introduction to RFID technology," IEEE Pervasive Computing, vol. 5, no. 1, pp. 25-33, 2006.

[13] E. S. Hochmair, "System optimization for improved accuracy in transcutaneous signal and power transmission," IEEE Transactions on Biomedical Engineering, vol. 31, no. 2, pp. 177-186, 1984.

[14] G. A. Kendir, W. Liu, G. Wang et al., "An optimal design methodology for inductive power link with Class-E amplifier," IEEE Transactions on Circuits and Systems I: Regular Papers, vol. 52, no. 5, pp. 857-866, 2005.

[15] M. Sawan, Y. Hu, and J. Coulombe, "Wireless smart implants dedicated to multichannel monitoring and microstimulation," IEEE Circuits and Systems Magazine, vol. 5, no. 1, pp. 21-39, 2005.

[16] R. F. Xue, K. W. Cheng, and M. Je, "High-efficiency wireless power transfer for biomedical implants by optimal resonant 
load transformation," IEEE Transactions on Circuits and Systems I: Regular Papers, vol. 60, no. 4, pp. 867-874, 2013.

[17] System Description-Wireless Power Transfer-Volume I: Low Power-Part 1: Interface Definition, Version 1.0.3, Wireless Power Consortium, 2011.

[18] C. Wang, O. H. Stielau, and G. A. Covic, "Design considerations for a contactless electric vehicle battery charger," IEEE Transactions on Industrial Electronics, vol. 52, no. 5, pp. 1308-1314, 2005.

[19] F. Broydé and E. Clavelier, "The emission level of medium-range inductive wireless power transmission systems," in Proceedings of the CEM International Symposium on Electromagnetic Compatibility, Session 1B, Rouen, France, April 2012.

[20] F. E. Terman, Radio Engineers'Handbook, McGraw-Hill, New York, NY, USA, 1943.

[21] J. A. Stratton, Electromagnetic Theory, McGraw-Hill, 1941.

[22] F. Broydé and E. Clavelier, "Maximum electric and magnetic field strengths at a given distance from some ideal antennas," IEEE Antennas \& Propagation Magazine, vol. 50, no. 6, p. 38, 2008, and "Correction", IEEE Antennas \& Propagation Magazine, vol. 50, no. 6, pp. 38, 2008.

[23] Council Recommendation 1999/519/EC of 12 July 1999 on the limitation of exposure of the genral public to electromagnetic fields ( $0 \mathrm{~Hz}$ to $300 \mathrm{GHz})$.

[24] EN 300 330-1 V1.7.1 (2010-02), Electromagnetic compatibility and Radio spectrum Matters (ERM); Short Range Devices (SRD); Radio equipment in the frequency range $9 \mathrm{kHz}$ to $25 \mathrm{MHz}$ and inductive loop systems in the frequency range $9 \mathrm{kHz}$ to $30 \mathrm{MHz}$; Part 1: Technical characteristics and test methods.

[25] EN 300 330-2 V1.5.1 (2010-02), Electromagnetic compatibility and Radio spectrum Matters (ERM); Short Range Devices (SRD); Radio equipment in the frequency range $9 \mathrm{kHz}$ to $25 \mathrm{MHz}$ and inductive loop systems in the frequency range $9 \mathrm{kHz}$ to $30 \mathrm{MHz}$; Part 2: Harmonized EN under article 3.2 of the R\&TTE Directive.

[26] EN 55011:2009 + Amendment A1:2010. Industrial, scientific and medical (ISM) radio-frequency equipment-Radio disturbance characteristics-Limits and methods of measurement.

[27] E. M. Thomas, J. D. Heebl, and C. Pfeiffer, "A power link study of wireless non-radiative power transfer systems using resonant shielded loops," IEEE Transactions on Circuits and Systems I: Regular Papers, vol. 59, no. 9, pp. 2125-2136, 2012.

[28] J. van Bladel, Electromagnetic Fields, Hemisphere Publishing Corporation, New York, NY, USA, 1985.

[29] "Guide to the R\&TTE Directive 1999/5/EC, Version of 20 April 2009," http://ec.europa.eu/enterprise/sectors/rtte/ documents/index_en.htm.

[30] "Technical assistance relating to the application of the EMC Directive and of the R\&TTE Directive-Specific Technical report no. 1-Emission of cochlear implants-Part B, 3rd Edition, May 13, 2005," Excem document 05032501D, http:// ec.europa.eu/enterprise/sectors/rtte/documents/guidance/index_en.htm. 

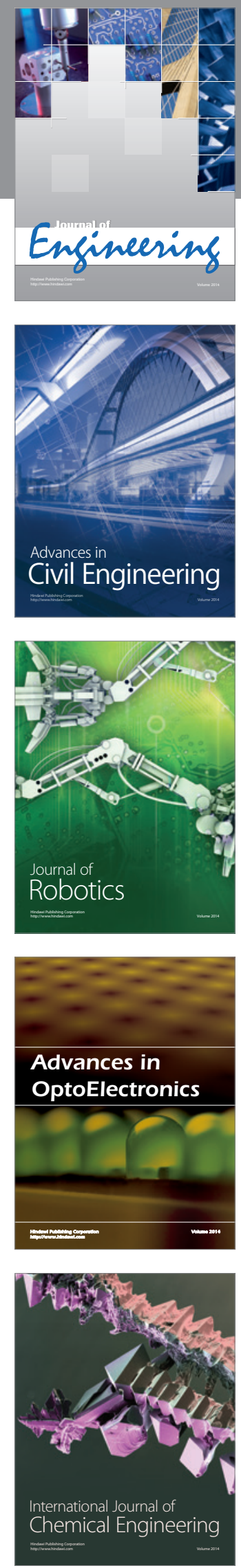

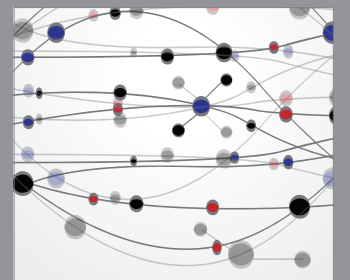

The Scientific World Journal
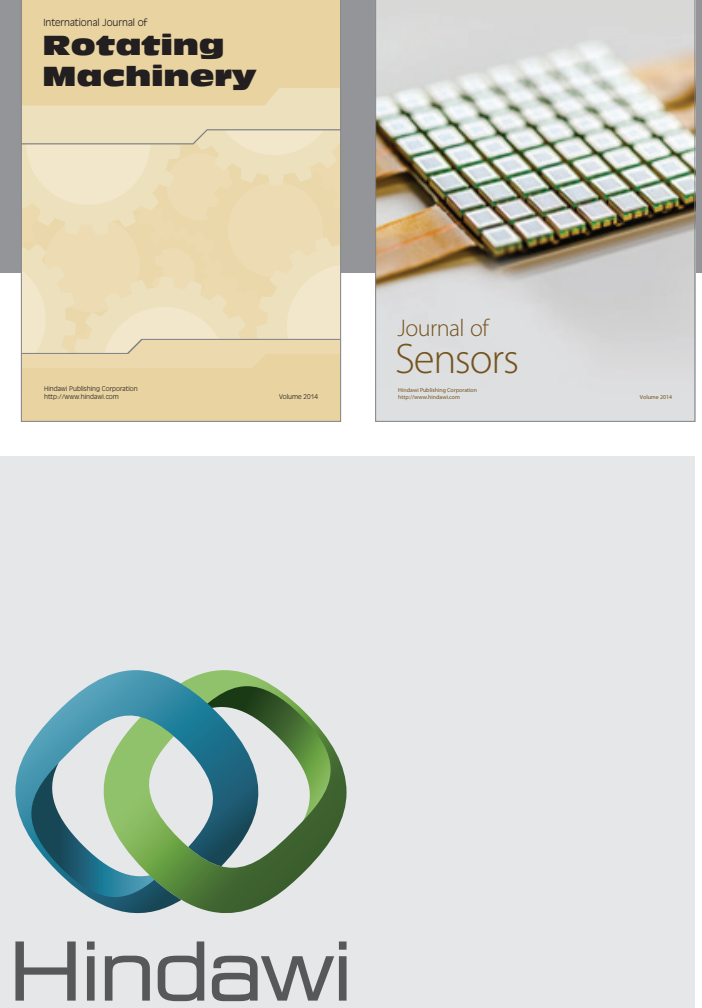

Submit your manuscripts at http://www.hindawi.com
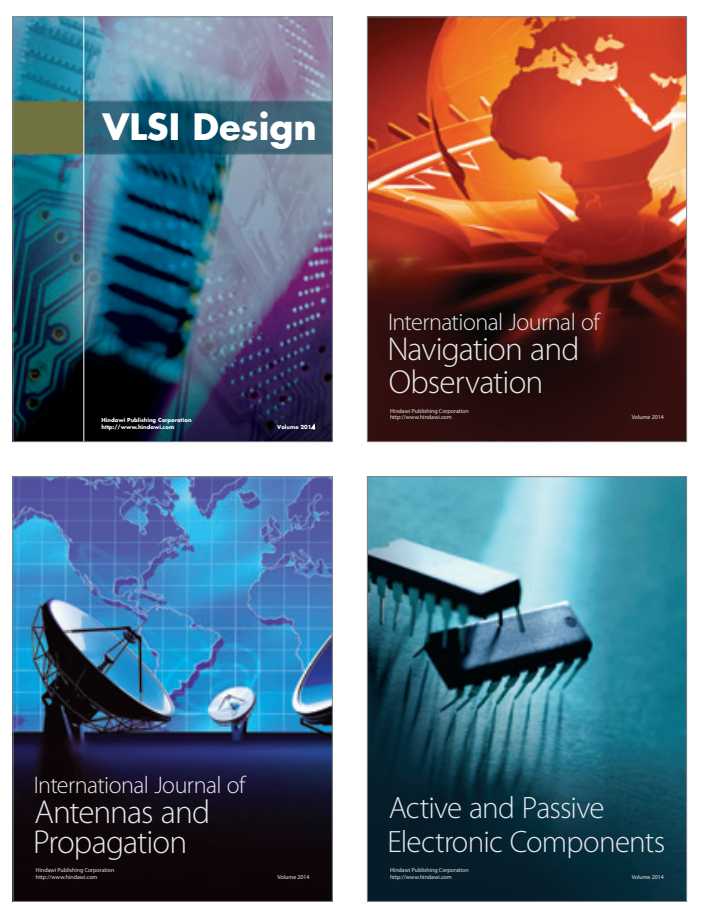
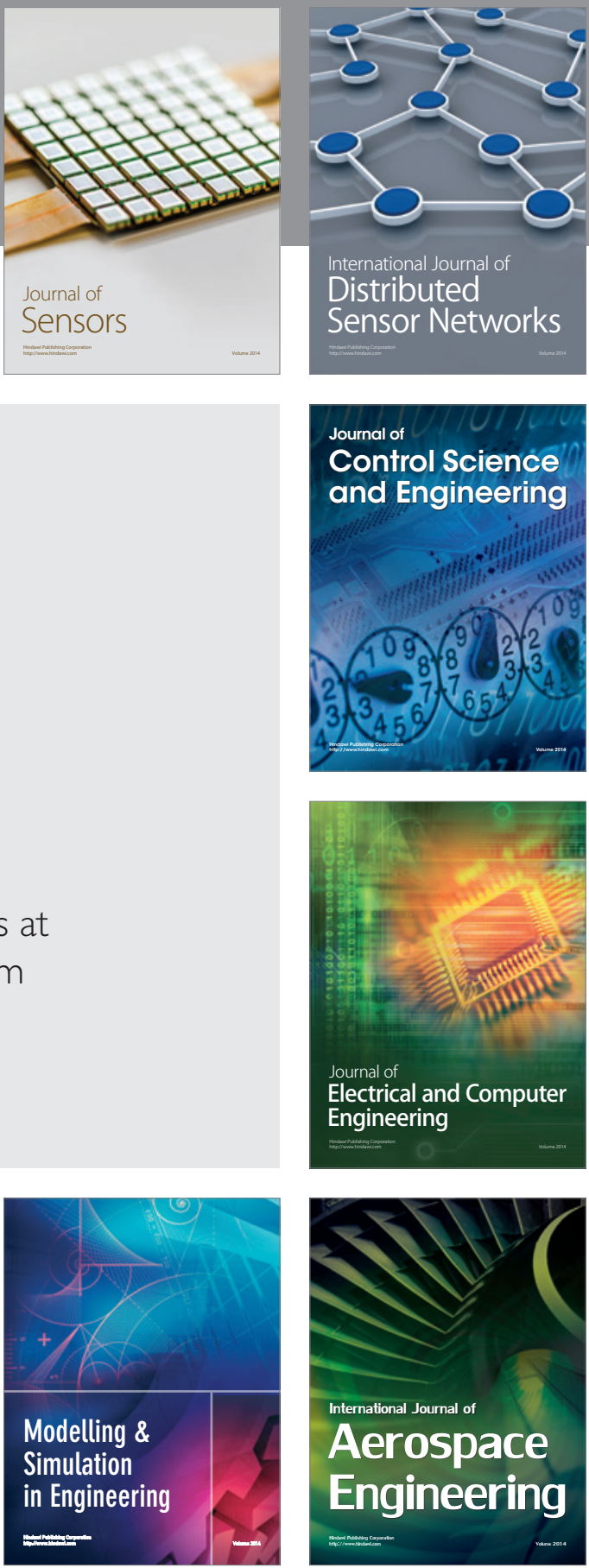

Journal of

Control Science

and Engineering
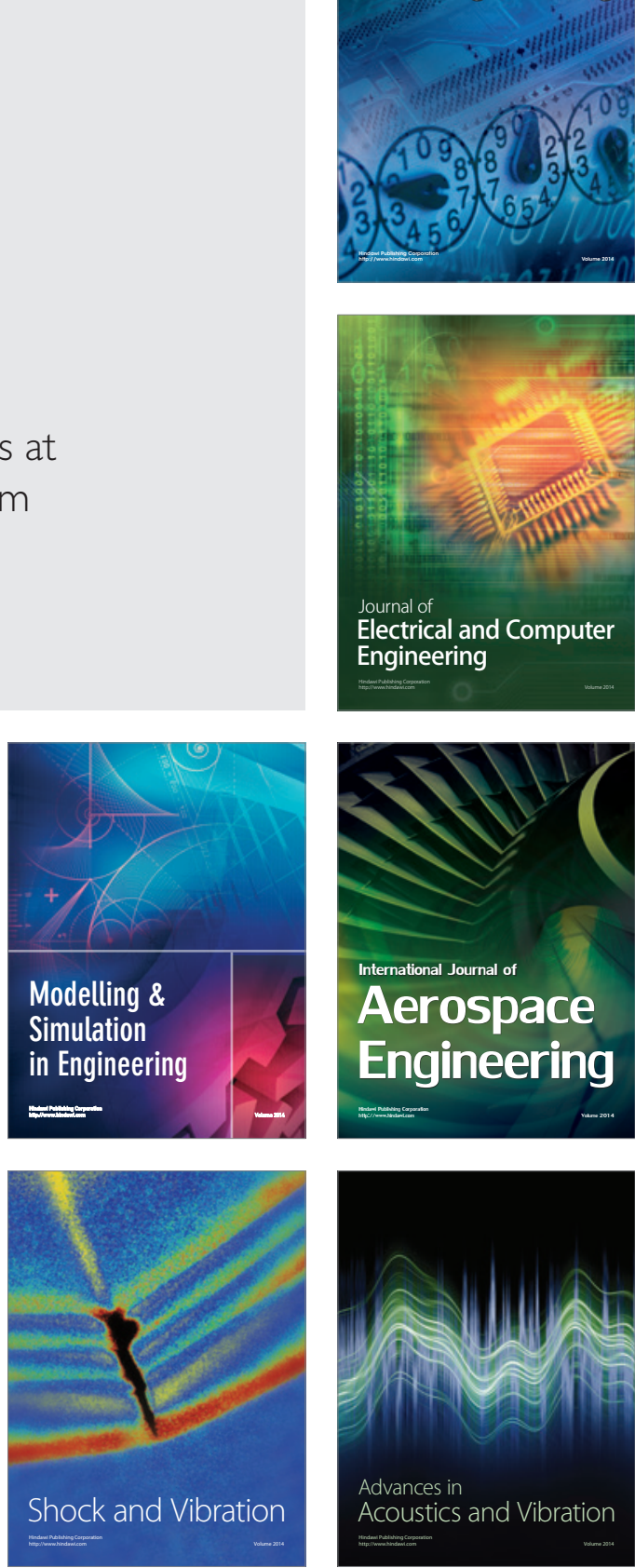